\title{
A CIB1-LIKE transcription factor GmCIL10 from soybean positively regulates plant flowering
}

\author{
YANG DeGuang $^{1 \dagger}, \mathrm{ZHAO}_{\mathrm{Wang}^{1 \dagger}}{ }^{\dagger}$ MENG YingYing ${ }^{2 \dagger}, \mathrm{LI}_{\mathrm{H}} \mathrm{Hong} \mathrm{Yu}^{2 *} \& \mathrm{LIU}^{2} \mathrm{Bin}^{2 *}$ \\ ${ }^{1}$ College of Agriculture, Northeast Agricultural University, Harbin 150030, China; \\ ${ }^{2}$ Institute of Crop Science, Chinese Academy of Agricultural Sciences, Beijing 100081, China
}

Received November 10, 2014, accepted January 6, 2015; published online February 3, 2015

\begin{abstract}
CRYPTOCHROME-INTERACTING basic helix-loop-helix 1 (CIB1) is a well characterized transcriptional factor which promotes flowering through the physical interaction with the blue light receptor CRYPTOCHROME 2 (CRY2) in Arabidopsis. However, the role of its counterpart in crop species remains largely unknown. Here, we describe the isolation and characterization of a CIB1 homolog gene, Glycine max CIB1-LIKE10 (GmCIL10), from soybean genome. The mRNA expression of GmCIL10 in the unifoliate leaves shows a diunal rhythm in both long day (LD) and short day (SD) photoperiod, but it only oscillates with a circadian rhythm when the soybean is grown under LDs, indicating that the clock regulation of GmCIL10 transcription is LD photoperiod-dependent. Moreover, its mRNA expression varies in different tissue or organs, influenced by the develpomental stage, implying that GmCIL10 may be involved in the regulation of multiple developmental processes. Similar to CIB1, GmCIL10 was evident to be a nuclei protein and ectopically expression of GmCIL10 in transgenic Arabidopsis accelerates flowering under both LDs and SDs, implying that CIBs dependent regulation of flowering time is an evolutionarily conserved mechanism in different plant species.
\end{abstract}

photoperiod, flowering time, soybean, Glycine max CIB1-LIKE10

Citation: Yang DG, Zhao W, Meng YY, Li HY, Liu B. A CIB1-LIKE transcription factor GmCIL10 from soybean positively regulates plant flowering. Sci China Life Sci, 2015, 58: 261-269, doi: 10.1007/s11427-015-4815-6

Cryptochrome is a type of blue light receptor regulating photomorphogenesis in plant and circadian clock in both plants and animals [1-4]. Plant genomes encode at least two kinds of cryptochrome: cryptochrome 1 (CRY1) and cryptochrome 2 (CRY2). In Arabidopsis, CRY1 primarily mediates blue light-dependent de-etiolation [5], while CRY2 majorly regulates photoperiodic flowering [6]. Arabidopsis CIB1, which is the first identified blue light-dependent CRY2-interacting protein, is a basic/helix-loop-helix (bHLH) transcriptional factor, acting as a CRY- and blue light-dependent regulator to stimulate floral initiation [7-9]. Hitherto, bHLH transcription factors have been well char-

$\dagger$ Contributed equally to this work

*Corresponding author (Email: liubin05@caas.cn, lihongyu@caas.cn) acterized to control a diversity of life processes in eukaryotes [9-12]. Members of this family contain a conserved bHLH signature domain, which consists of two functionally distinct regions. The basic region is involved in binding to E-box (CANNTG), and the HLH region functions as a dimerization domain [13-16]. CIB1 binds to the G-box (CACGTG), a canonical E-box, in vitro [7]. However, CIB1 can form heterodimers with other CIB1-related proteins and targets to the no-canonical E-box (CANNTG) of the FLOWERING LOCUS T (FT) promoter in vivo to regulate transcription [9]. CIBs function in mediating CRY2-CIB signal transduction pathway to activate floral initiation [7-9]. CRY2-CIB blue light-dependent interaction has also been tested in soybean (Glycine max). GmCIB1 interacts 
with the E-box-containing promoter sequences of the senescence-associated gene WRKY DNA BINDING PROTEIN $53 b(W R K Y 53 b)$ to activate leaf senescence, whereas photo-excited GmCRY2a physically interacts with GmCIB1 to suppress its DNA binding activity and GmCRY2a acts antagonistically with GmCIB1 to mediate light suppression of leaf senescence in soybean [17]. These findings indicated that CRY-CIB signaling mechanism is evolutionarily conserved in plants, although this mechanism in different plant species may mediate various aspects of plant development in response to light.

Flowering promotion is a major physiological function of CIB1 in Arabidopsis which is a primary model organism for the study of photoreceptor signal transduction. However, whether CIB-related proteins are involved in the flowering time regulation in crops is hitherto unclear [18]. In this study, we isolated a CIBI homolog gene GmCIL1O in soybean, and analyzed its expression profiles in response to photoperiod or in various tissues at different development stages. Moreover, we investigated the subcellular localization of GmCIL10 protein in Arabidopsis protoplasts and evaluated its role in flowering time regulation through ectopic overexpression of GmCILIO in Arabidopsis. These results provided support a hypothesis that GmCIL10 in soybean is a positive regulator of flowering time and CIB mediated flowering time regulation is a conserved mechanism in plant species.

\section{Materials and methods}

\subsection{Plant materials and growth conditions}

An elite soybean (Glycine max) cultivar Ken-nong 18 (KN18) was cultured in environmentally controlled growth rooms with a defined photoperiod, short day (SD, $8 \mathrm{~h}$ light/16 h dark) or long day (LD, $16 \mathrm{~h} \mathrm{light/} 8 \mathrm{~h}$ dark), at 25 to $28^{\circ} \mathrm{C}$. Cool white fluorescent lights (TLD $18 \mathrm{~W} / 54$, Philips) were used as a white light source ( 200 to 300 $\mathrm{mmol} \mathrm{m} \mathrm{m}^{-2}$ above the plant canopy). Samples were collected as described before with some modifications [19-23]. For spatial and temporal expression analysis, the plants were grown under SDs and all samples were collected $0.5 \mathrm{~h}$ after light on. The unifoliolates, and the first to fourth trifoliolates were collected at indicated growth stages. Developing seeds were collected at 7,14 and 21 days after flowering and the mature seeds were collected when the seeds became yellow. For the circadian rhythm analysis, the fully expanded unifoliolates of the 2-week-old soybeans were sampled every $2 \mathrm{~h}$ for 2 days under LD or SD photoperiod and for additional 2 days in continuous light or dark. At least five individual plants per sample were harvested and all samples were immediately frozen in liquid nitrogen and stored at $-80^{\circ} \mathrm{C}$ before being analyzed. All experiments were repeated three times under consistent conditions.

\subsection{RNA isolation, gene cloning and vector construc- tion}

Total RNA was extracted by Trizol reagent (Invitrogen, USA) and cDNA synthesis was performed according to Revert Aid first-strand cDNA synthesis kit manual (Promega, USA). The Glymal16g10820 (GmCIL10) coding DNA sequence (CDS) was amplified using the primer pair GmCIL10-F and GmCIL10-R (Table 1), and then cloned into the pGWC vector [24]. The ectopic expression vector pLeela-GmCIL1O and the subcellular localization vector pENSG-YFP-GmCIL10 were constructed respectively by LR recombination reaction (Invitrogen, USA). The recombinant protein expression vector pCold-GmCIL10 was constructed by insertion of GmCIL1O CDS into the pCold $^{\mathrm{TM}} \mathrm{TF}$ DNA (TaKaRa, Japan) vector at the Xhol restriction site by In-fusion recombination reaction (Clontech, USA).

\subsection{Bioinformatics analysis}

The alignment of amino acid sequence was performed using Clustal W software with default parameters. The Neighbor-joining phylogenetic tree was constructed by MEGA software (version 4.0) using bootstrap analysis (1000 replicates). The schematic diagrams were constructed using Adobe Illustrator CS6 software, based on the genome sequences from the database (http://www.phytozome.net/ Glycine max v1.1).

\subsection{Real-time quantitative reverse transcription PCR}

Expression profiles were investigated by Real-time quantitative reverse transcription PCR (RT-qPCR), using SYBR Premix Ex Taq polymerase kit (TaKaRa, Japan) and ABI StepOne Real-Time PCR system (Applied Biosystems, USA). Each $15-\mu \mathrm{L}$ reaction system consisted of $5 \mu \mathrm{L}$ template, 7.5 $\mu \mathrm{L} 2 \mathrm{X}$ SYBR Premix, $200 \mathrm{nmol} \mathrm{L}^{-1}$ of each primer, and $0.3 \mu \mathrm{L}$ ROX. The specific primers used for the quantification of GmCIL10 GmFT2a, GmFT5a, and the reference genes were listed in Table 1 . The relative expression unit (REU) was calculated following the formula: $\mathrm{REU}=2^{(\mathrm{Cta}-\mathrm{Ctb})}$, where Cta and $\mathrm{Ctb}$ are the average $\mathrm{Ct}$ (Cycle threshold) values of the reference and target genes respectively [25]. Each experiment was repeated at least three times.

\subsection{Subcellular localization analysis}

The subcellular localization vector pENSG-YFP-GmCIL10 and the nuclear-marker vector pENSG-CFP-AHL22 were co-transformed into Arabidopsis mesophyll protoplasts following the previous procedure [26, 27]. The protoplast transformed with pENSG-YFP vector was used as control. The transformed protoplasts were incubated in dark for $12-14 \mathrm{~h}$ at $23^{\circ} \mathrm{C}$, and then analyzed under a confocal mi- 
Table 1 The sequence of Primers used in this study

\begin{tabular}{ll}
\hline Primer Name & Sequence (5' to 3 $\left.^{\prime}\right)$ \\
\hline GmCIL10-F & ATGGAAAACCAGTTCTTTCTGGCTT \\
GmCIL10-R & TCAGAGCTCAACTTTCATCTGTG \\
GmCIL10-pCold-F & TCAAGCTTGTCGACCTGCAGATGGAAAACCAGTTCTTTCTGGCTT \\
GmCIL10-pCold-R & ATTACCTATCTAGACTGCAGTCAGAGCTCAACTTTCATCTGTG \\
GmCIL10-QF & CTTCTCAACTCCAACCCAATCAAC \\
GmCIL10-QR & GTAGCAGTAGCAATGTGGCAAAG \\
GmACT11-F & ATCTTGACTGAGCGTGGTTATTCC \\
GmACT11-R & GCTGGTCCTGGCTGTCTCC \\
GmSKIP16-F & GAGCCCAAGACATTGCGAGAG \\
GmSKIP16-R & CGGAAGCGGAAGAACTGAACC \\
GmUKN1-F & TGGTGCTGCCGCTATTTACTG \\
GmUKN1-R & GGTGGAAGGAACTGCTAACAATC \\
pLeela-F & GTTATGGGTCAACGGTTTC \\
ACT2-F & AAGCTCTCCTTTGTTGCTGTT \\
ACT2-R & GACTTCTGGGCATCTGAATCT \\
GmFT2a-QF & GGTTCTGGTGGAAGGAGGTTATAC \\
GmFT2a-QR & ACTACTAAAGAGTGTGGGAGATTGC \\
GmFT5a-QF & GGACAGAAGCAAAATTAAAGCAGATG \\
GmFT5a-QR & ACTATATACTATGATGTTTGTGTTGGG \\
E-box-F & TCACTCATTAGGGCAAACTGTGGGTCAATAATCAGGAACTAGGAGAGTGG \\
E-box-R & AGGGCCTGAGATCGGCTTGTAATCGCGCAATGCAAAAGAGCGCANNTGGCCCACTCTCCTAGTTCCTGAT \\
G-box-R & AGGGCCTGAGATCGGCTTGTAATCGCGCAATGCAAAAGAGCGCACGTGGCCCACTCTCCTAGTTCCTGAT \\
Em-box-R & AGGGCCTGAGATCGGCTTGTAATCGCGCAATGCAAAAGAGCGAAAAAAGCCCACTCTCCTAGTTCCTGAT \\
E-box-QF & CATTAGGGCAAACTGTGGGTC \\
E-box-QR & CCTGAGATCGGCTTGTAATC \\
\hline
\end{tabular}

croscope (Leica TCS SP2).

\subsection{Transgenic Arabidopsis preparation and pheno- type analysis}

Agrobacterium tumefaciens strain GV3101 (pMP90RK) harboring pLeela-GmCIL1O vector was used for the transformation of Col-4 Arabidopsis ecotype through the floral dip method. The transgenic lines were screened using 100 $\mathrm{mg} \mathrm{\textrm {L } ^ { - 1 }}$ glufosinate ammonium and further confirmed by PCR using the primers (GmCIL10-R and pLeela-F) specific to GmCIL1O gene and pLeela vector backbone (Table 1). The mRNA expression analysis of GmCIL10 in the transgenic lines was performed using the primers (GmCIL10-QF and GmCIL10-QR) specific to GmCIL10 CDS (Table 1). T2 plants were grown under LDs or SDs in growth chambers for the measurement of flowering time and leaf numbers as described before [26].

\subsection{Quantitative PCR-based DNA binding assay}

The quantitative PCR-based DNA binding assay experiments were performed as previously described with minor modifications [17]. The CDS of GmCIL10 was inserted into pCold $^{\mathrm{TM}} \mathrm{TF}$ vector and the recombinant construct was transformed into E.coli (BL21). The expression of recombinant protein His-TF-GmCIL10 was induced. The E.coli lysate expression the His-TF-GmCIL10 protein or the
His-TF control protein was prepared respectively in lysis buffer [50 mmol L ${ }^{-1}$ Tris ( $\mathrm{pH} 7.8$ ), $500 \mathrm{mmol} \mathrm{L}^{-1} \mathrm{NaCl}$, $0.5 \%$ Triton X-100, 1 mmol L ${ }^{-1}$ PMSF, 5 mmol L ${ }^{-1}$ DTT, 1 tablet $/ 50 \mathrm{~mL}$ of protease inhibitor cocktail], mixed with Dynabeads His-Tag Isolation \& Pulldown (\#10104D, Novex) for $15 \mathrm{~min}$ at room temperature, and then washed six times with the wash buffer $\left(50 \mathrm{~m} \mathrm{~mol} \mathrm{~L}^{-1}\right.$ Tris, $\mathrm{pH} 7.8$, $500 \mathrm{~m} \mathrm{~mol} \mathrm{~L}^{-1} \mathrm{NaCl}, 0.1 \%$ Triton $\mathrm{X}-100$, and $1 \mathrm{mmol} \mathrm{L}^{-1}$ PMSF) and collected with the DynaMag ${ }^{\text {TM }}$-Spin apparatus (Novex, USA). His-TF-GmCIL10 or His-TF protein binding with dynabeads was collected and diluted to $0.5 \mu \mathrm{g} \mu \mathrm{L}^{-1}$, with the concentration determined using the Bradford method. The Ewt, Gwt and Em DNA sequences were prepared by the annealing and extending reactions with respective primer pairs (Table 1): E-box-F/E-box-R for Ewt, E-box-F/G-box-R for Gwt, and E-box-F/Em-box-R for Em. Each reaction consisted of $25 \mu \mathrm{L} 2 \mathrm{X}$ Prime STAR GC Buffer, $10 \mu \mathrm{L}\left(10 \mu \mathrm{mol} \mathrm{L}{ }^{-1}\right)$ of each primer, $4 \mu \mathrm{L}$ dNTP Mixture, 0.5 $\mu \mathrm{L}$ Prime STAR HS DNA Polymerase (TaKaRa). PCR reaction was programmed as following: $100^{\circ} \mathrm{C}, 2 \mathrm{~min}$; Slowly ramp to $45^{\circ} \mathrm{C}$, and hold on for $10 \mathrm{~min}$; $72^{\circ} \mathrm{C}, 10 \mathrm{~min}$. Each $15-\mu \mathrm{L}$ DNA binding reaction consisted of $5 \mu \mathrm{L}$ dynabeads, $0.4 \mu \mathrm{mol} \mathrm{L} \mathrm{L}^{-1}$ DNA, $2.5 \mu \mathrm{L} 5 \mathrm{X}$ DNA binding buffer [20\% Glycerol, $2.5 \mathrm{mmol} \mathrm{L}^{-1}$ DTT, 250 $\mathrm{mmol} \mathrm{L}^{-1} \mathrm{KCl}, 1 \mathrm{mg} \mathrm{mL}^{-1} \mathrm{BSA}, 50 \mathrm{mmol} \mathrm{L}^{-1}$ Tris-HCl (pH 7.5), $5 \mathrm{mmol} \mathrm{L}{ }^{-1} \mathrm{MgCl}_{2}$ ], kept in room temperature for 15 min, and then washed six times with $1 \mathrm{X}$ DNA binding 
buffer. The precipitated products were eluted with $50 \mu \mathrm{L}$ elution buffer [50 mmol L ${ }^{-1}$ Tris- $\mathrm{HCl}$ (pH 7.8), $300 \mathrm{mmol}$ $\mathrm{L}^{-1} \mathrm{NaCl}, 300 \mathrm{mmol} \mathrm{L}^{-1}$ Imidazole ( $\mathrm{pH} 7.5$ )], and diluted 50-fold prior to quantitative PCR analysis using primer pairs E-box-QF/E-box-QR (Table 1). The DNA binding activity was calculated by the formula: $2^{-\mathrm{Ct}}$. Ct (cycle threshold) represents the number of cycles required for the fluorescence signal to exceed background level.

\section{Results}

\subsection{GmCIL10 is a member of the bHLH transcription factor family}

We analyzed the soybean (Glycine max cv. Williams 82) genome deposited in the Phytozome database (http://www. phytozome.org) and identified 33 homologous proteins shared conserved bHLH domain with AtCIBs or AtCILs (AtCIB1-LIKEs). Phylogenetic analysis indicated that these proteins were classed into two groups and that bHLH proteins in Group I are more closely related to AtCIB1 than those proteins in Group II (Figure 1A). The protein encoded by Glymal11g12450 locus was previously designed as GmCIB1 because it is evolutionally close to AtCIB1 in the phylogenetic tree and has been experimentally proofed to interact with GmCRY2a [17]. To keep consistent, other bHLH proteins in Group I were designed as GmCIL1 (GmCIB1-LIKE1) to GmCIL13 respectively, according to their relative evolution distance to GmCIB1.

GmCIL10, encoded by locus Glymal16g10820, belongs
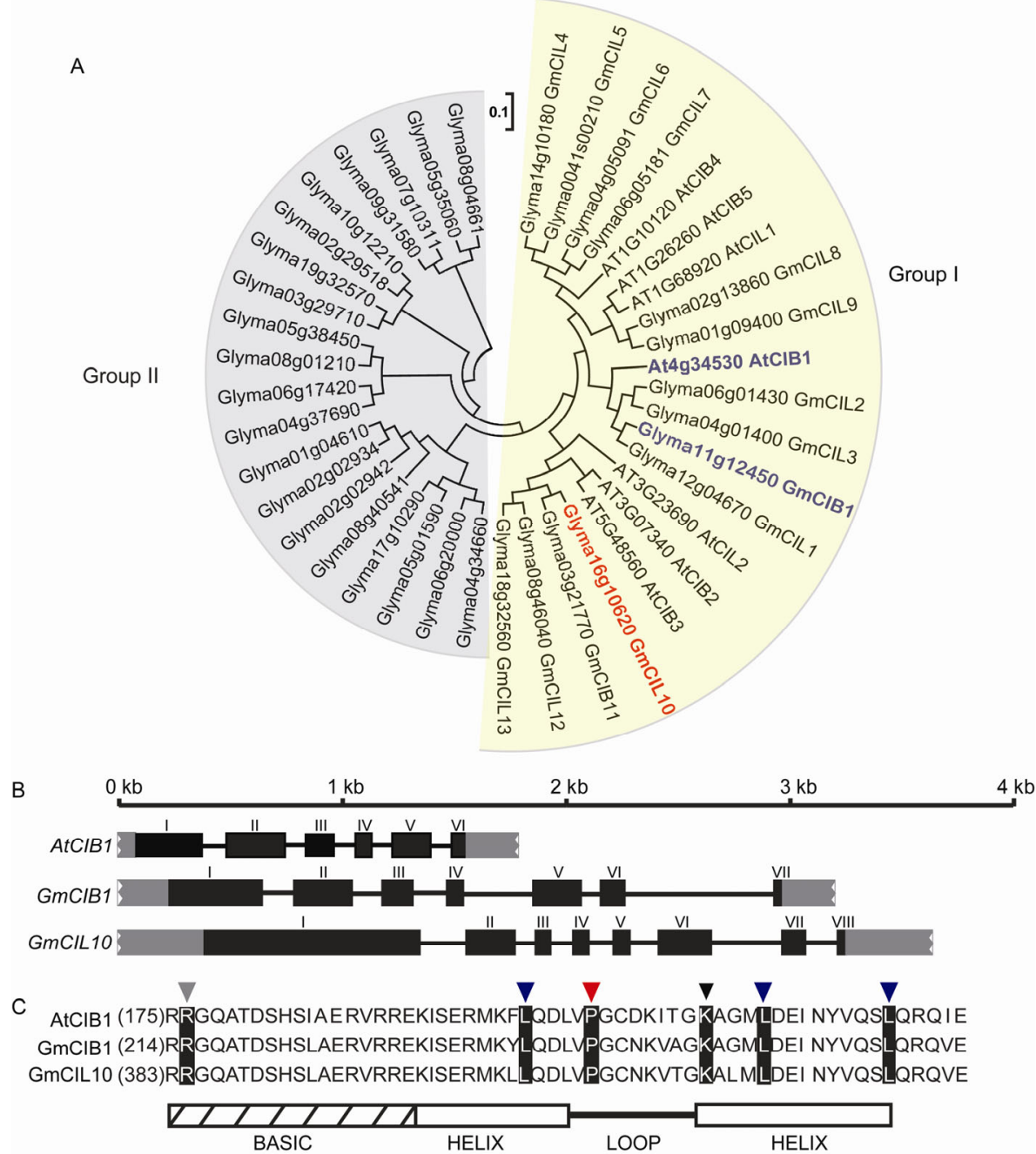

Figure 1 (color online) Phylogenetic analysis and comparisons of the gene structures. (A) Phylogenetic tree demonstrating the evolutionary relationships between Arobidopsis CIBs and soybean homologous proteins. Soybean protein sequences were downloaded from Phytzome V9.1 (http://www. phytozome.net). At, Arabidopsis thaliana; Gm, Glycine max. The scale bar indicates substitutions per site (1,000 replicates). (B) Diagram representation of gene structures of AtCIB1, GmCIB1 and GmCIL10. The numbers on the top of the line segments indicate the length of the DNA sequences.The black boxes represent exons; The lines represent introns; The grey boxes represent UTRs. (C) Alignment of the amino acid sequences (by ClustalX with a manual adjustment) of bHLH domains of AtCIB1, GmCIB1 and GmCIL10. The key amino acids of bHLH domain (black) are shown. A diagram depicting the boundaries of basic and helix- loop-helix motifs is included at the bottom. 
to a sub-group which is least close to the sub-group of AtCIB1 and GmCIB1 within group I. We were curious whether GmCIL10 is functionally conserved or not to AtCIB1 or GmCIB1. Therefore, we cloned GmCIL10 coding DNA sequence (CDS) from cv. Kennong18 (KN18), which encodes a 590-residue polypeptide. We aligned the CDSs of AtCIB1, GmCIB1, and GmCILIO with their genomic DNA sequences (Figure 1B). The result showed that the genomic sequence of GmCILIO is the largest and AtCIBI is the smallest among the three genes. Besides, GmCIL10 gene consists of 8 exons while $A t C I B 1$ and GmCIBI comprise 6 and 7 exons respectively. Although their gene structures are different from each other, peptide alignment of their bHLH domains demonstrated that the amino acid sequences are highly conserved, especially those representing residues of the BASIC, HELIX, LOOP or HELIX motifs, which are completely consistent (Figure 1C), implying these proteins may share conserved function. To test this hypothesis, we analyzed if GmCIL10 is able to bind E-box (CANNTG) cis-element like many other bHLH transcriptional factors [7]. The results demonstrated that GmCIL10 interacted with a higher affinity in vitro to E-box, and especially to a canonical E-box, name G-box (CACGTG) (Figure 2).

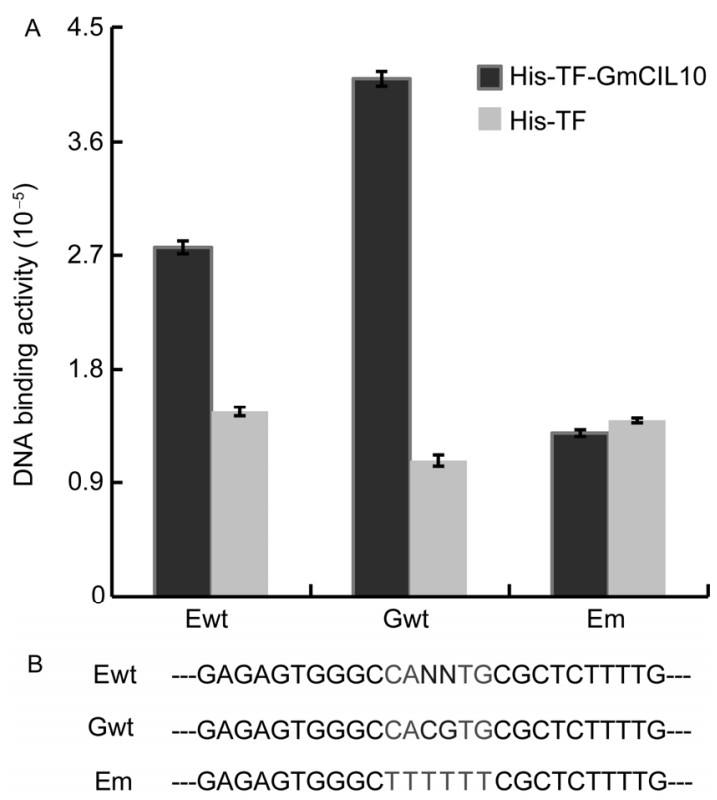

Figure 2 GmCIL10-DNA interaction in vitro. (A)The DNA binding assay shows the interaction of GmCIL10 protein with wild-type E-box (Ewt), G-box (Gwt) or mutant E-box (Em) DNA sequence. His-TF-GmCIL10 fusion protein or His-TF protein (used as control) expressed in E.coli BL21 was purified using Dynabeads His-Tag Isolation \& Pulldown kit, then incubated with the Ewt, Gwt or Em DNA fragment for $15 \mathrm{~min}$ at room temperature. After six times of washing with $1 \mathrm{X}$ binding buffer, the bound DNA was eluted by the elution buffer and subjected to quantitative PCR. The DNA binding activity was calculated as in method. standard deviation (SD, $n=3$ ) was shown. (B) The DNA sequences of Ewt, Gwt and Em used in (A) was shown.

\subsection{Diurnal or circadian expression pattern of GmCILIO in response to photoperiod}

A previous study reported that GmCRY1a is a major regulator of photoperiodic flowering in soybean and the oscillated abundance of GmCRY1a protein significantly correlates with latitudinal distribution of soybean cultivars and their photoperiodic flowering time [22]. Taking that CIB1 physically interact with CRY2 in a blue light specific manner and promotes flowering in Arabidopsis, we reasoned that if GmCIL shows a similar rhythmic expression patterns as GmCRY1a, it may coordinate with GmCRY1a in flowering time regulation in soybean. To test this hypothesis, we analyzed the mRNA expressions of GmCIL genes and found that $G m C I L 10$ is expressed in a photoperiod-dependent manner. In this experiment, we grew soybean in short days (SDs, $8 \mathrm{~h}$ light/16 h dark) or long days (LDs, $16 \mathrm{~h}$ light $/ 8 \mathrm{~h}$ dark) for two weeks until the unifoliolates are fully developed, and collected the unifoliolates every $2 \mathrm{~h}$ for two successive $24 \mathrm{~h}$ cycles. The plants were then transferred to continuous light (LL) or continuous darkness (DD), and the samples were collected every $2 \mathrm{~h}$ for additional $48 \mathrm{~h}$. RT-qPCR results showed that, under SDs, the GmCIL10 mRNA oscillated with a diurnal rhythm with a peak $4 \mathrm{~h}$ after dawn (ZT4) (Figure 3A and B). Such a diurnal expression pattern is similar to that of GmCRYla mRNA [22], implying that GmCIL10 may coordinate with GmCRY1a to regulate photoperiodic flowering. However, the oscillation of GmCIL10 mRNA became random in subsequent LL or DD (Figure 3A and B), and the amplitude increased in LL (Figure 3A) but decreased in DD (Figure 3B). In contrast to that under SDs, GmCIL10 mRNA under LDs oscillated with a diunal rhythm with a peak $4 \mathrm{~h}$ after dusk and such a rhythm was well maintained in LL or DD (Figure 3C and D), although the amplitude decreased in DD, demonstating that the circadian oscillation of GmCILIO mRNA is LD-photoperiod dependent. It's interesting that GmCILIO expression peaking at different time under SDs and LDs. Such kind of inconsistent gene expression pattern has been observed in photoperiodic flowering regulation which is mediated by co-operation between internal circadian clocks and external environmental stimuli, like diurnal photoperiod. For example, the transcription level of CONSTANS $(\mathrm{CO})$, the key regulator determining the photoperiodic flowering in Arabidopsis, peaks and fluctuates differently in LDs and SDs. It has been illustrated that transcriptional regulation of $C O$ gene by the interaction between circadian clock and environmental photoperiod is the most crucial mechanisms for day-length measurement in photoperiodic flowering of Arabidopsis. The inconsistent expression patterns of GmCIL10 under LDs and SDs imply that internal and external signals may co-regulate GmCILIO expression throught multiple transcriptional regulators, and GmCILIO may play important role in modulating photoperiodic flowering or other life history trait. 

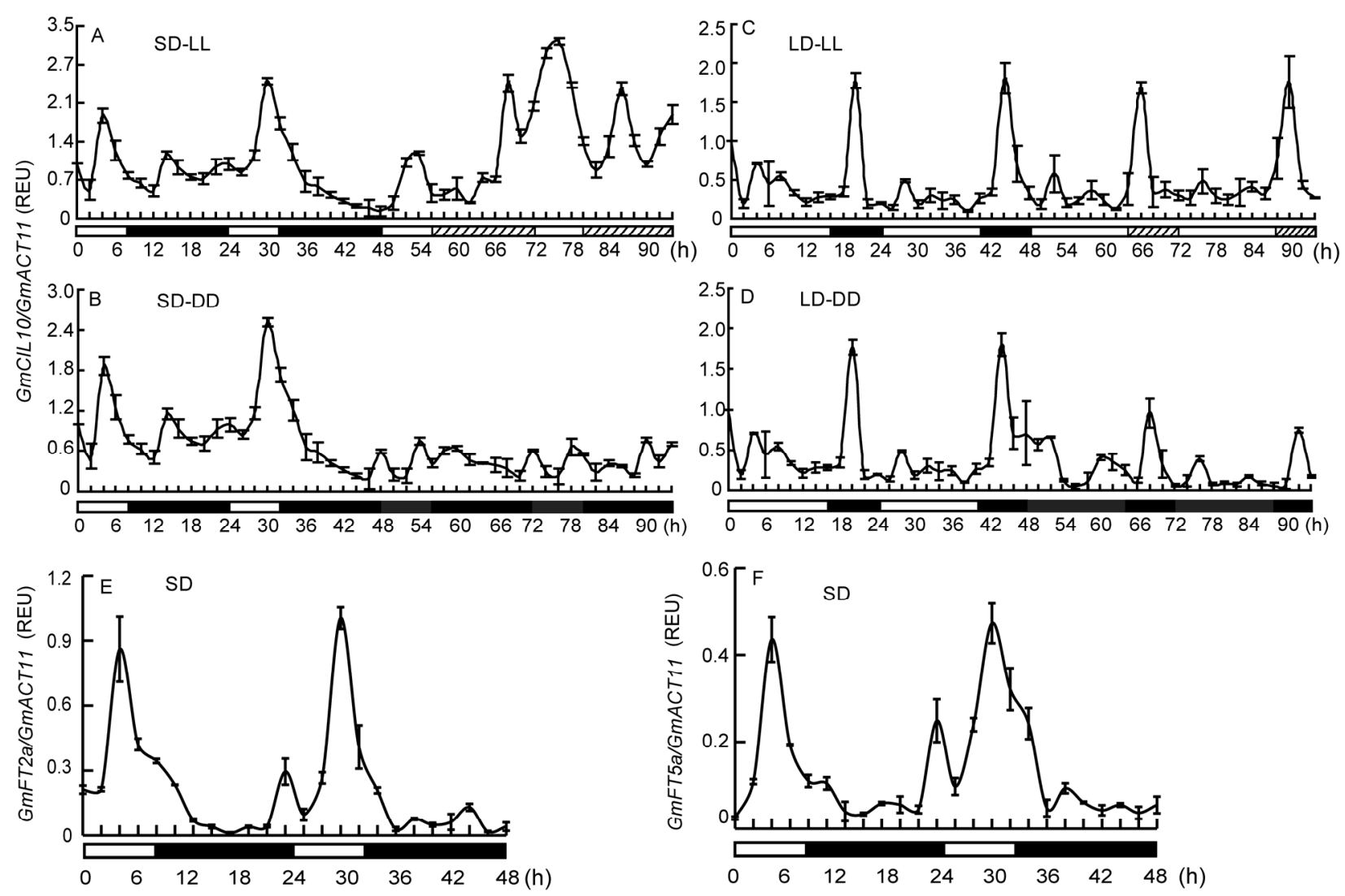

Figure 3 Quantitative PCR (RT-qPCR) showing mRNA expressions in response to different photoperiod treatments. Unifolioate leaves were collected every $2 \mathrm{~h}$ as indicated. White box, objective light phase; Dark box, objective dark phase; Striped box, subjective dark phase; Gray box, subjective light phase. (A to D) mRNA expressions of GmCIL10. (A and B) Soybean plants grown under short day photoperiods (SDs) for 14 days were transferred to continuous white light (LL) (A) or continuous dark (DD) (B) for 2 days. (C and D) Samples grown under long day photoperiods (LDs) were treated and collected as in (A and B). (E and F) mRNA expressions of GmFT2a (E) or GmFT5a (F) under SDs.

\subsection{Spatial and temporal expression patten of GmCILIO}

FLOWERING LOCUS T (FT) is a key flowering integrator that encodes florigen in Arabidopsis. Two of the FT homologs, GmFT2a and GmFT5a, were reported to coordinately control flowering time in soybean [28]. Similar to GmCIL10 and GmCRYIa, GmFT2a and GmFT5a show diurnal expression patterns with a peak $4 \mathrm{~h}$ after dawn under SDs (Figure 3E and F). Taking that CIB1 and CRY2 physically interact and directly promote $F T$ transcription in Arabidopsis, we surmised that GmCIL1O may associate with GmCRYIa to up-regulate GmFT2a and GmFT5a, and then trigger flowering in soybean. To test this possibility, we investigate the spatial and temporal expression profile of GmCILIO in the unifolioates and trifoliolates of soybeans for a consecutive growth stages grown under SDs (Figure 4). To avoid improper interpretation of its expression levels in various tissues at different stages, RT-qPCR was performed using two stably expressed reference genes, GmSKIP16 (Figure 4A) and GmUKNI (Figure 4B) [29]. The levels of GmCIL1O transcripts in the unifolioates and trifoliolates under SDs increased gradually to their maximum levels prior to the initiation of flowering, and then decreased sharply when the time of flowering approached (Figure 4). Such an expression feature of GmCIL10 mRNA resembles that of GmFT2a or GmFT5a in soybeans grown under SDs [28]. In addition, we observed that the level of GmCIL1O transcripts increased gradually with the development of the seed, reaching a peak 21 days after pod initiation and thereafter decreased at maturation (Figure 4), indicating GmCIL10 may also play a role during pod development.

\subsection{Subcellular localization of GmCIL10 protein}

To investigate the subcellular compartment where GmCIL10 functions, YFP-GmCIL10 fusion protein was transiently expressed in Arabidopsis protoplasts, and then analyzed by YFP fluorescence under the confocal microscopy (Leica TCS SP2). The result showed that YFPGmCIL10 protein was co-localized with the nuclear protein CFP-AHL22 in the nucleus (Figure 5A), while the YFP protein alone present in both nucleus and cytoplasm (Figure 5B) [26].This result suggests that GmCIL10 may function as a transcription factor. 

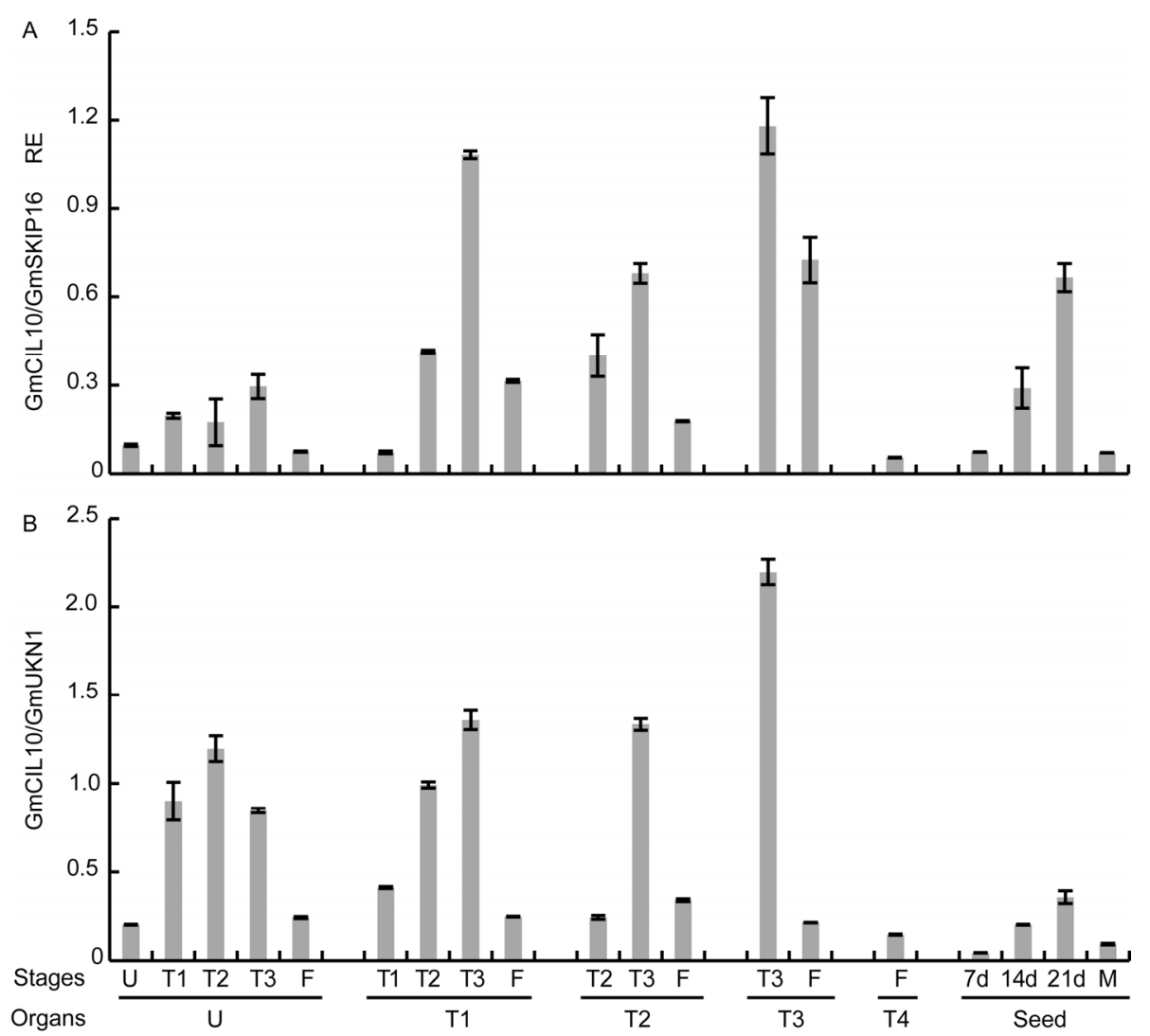

Figure 4 The mRNA expression profiles of GmCIL10 in leaves or seeds at different growth stages. The indicated samples were collected from the plants grown in SDs at different developmental stages. Organs: U, unifoliolates; T1 to T4, the 1st to 4th trifoliolates. Stages: U, T1,T2, T3 or T4 indicates the time when the unifoliolates or the first to fourth trifoliolates are fully opened respectively; F, flowering; $7 \mathrm{~d}, 14 \mathrm{~d}$, and $21 \mathrm{~d}$ indicate the days after flowering respectively; M indicated the seed mature stage. The mRNA level of GmSKIP16 (A) or GmUKN1 (B) was used as the internal control respectively. The RT-qPCR results shown are average $( \pm \mathrm{SD})$ of three biological repeats.
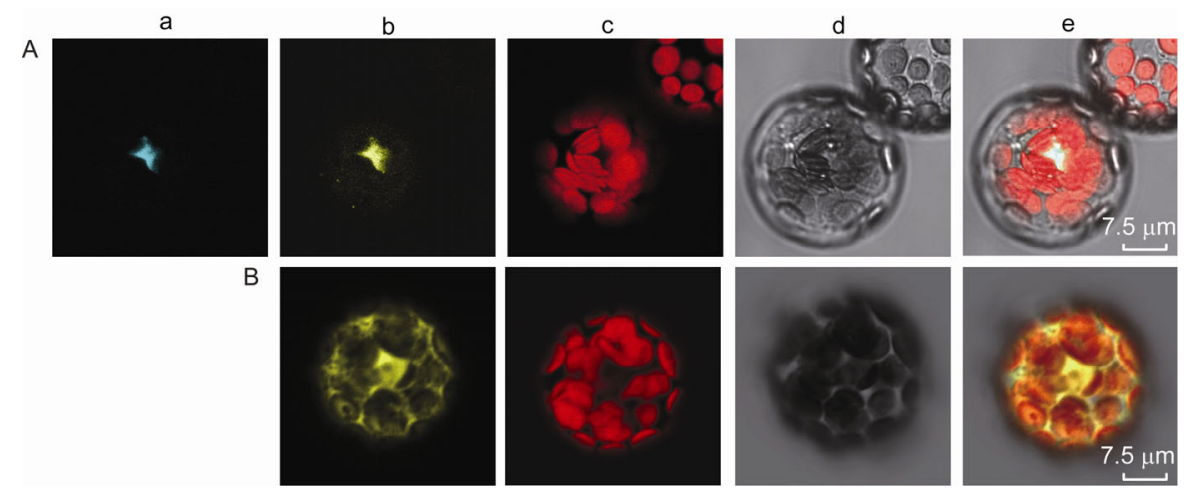

Figure 5 (color online) Subcellular localization of GmCIL10 protein in Arobidopsis mesophyll protoplasts. (A) GmCIL10 protein co-localized with a nuclear protein AHL22 in nucleus of mesophyll protoplasts. The plasmids encoding the YFP-GmCIL10 or CFP-AHL22 protein were co-transformed into the mesophyll protoplasts of 4-week-old plants grown under LDs , incubated for 12-14 h prior to the confocal microscope analysis. Image a, CFP fluorescence; Image b, YFP fluorescence; image c, Auto fluorescence; image d, Bright field; image e, Merge of images from a to d. Bar, $7.5 \mu \mathrm{m}$. (B) The images of protoplast expressing YFP protein alone were used as control.

\subsection{Ectopic expression of GmCIL10 promotes flower- ing in Arabidopsis}

To investigate if GmCIL10 has an activation activity in floral initiation, we prepared transgenic Arabidopsis that expressed the 35S:GmCIL10 transgene. Multiple transgenic lines showed similar early flowering time phenotype under both LDs and SDs (Figure 6A and B, two independent lines were shown). In consistent with the early flowering phenotype, the ectopic overexpression of GmCILIO in three independent lines (OX-1, OX-2 and OX-3) was verified by RT-qPCR (Figure 6C). Statistical analysis indicated that the 

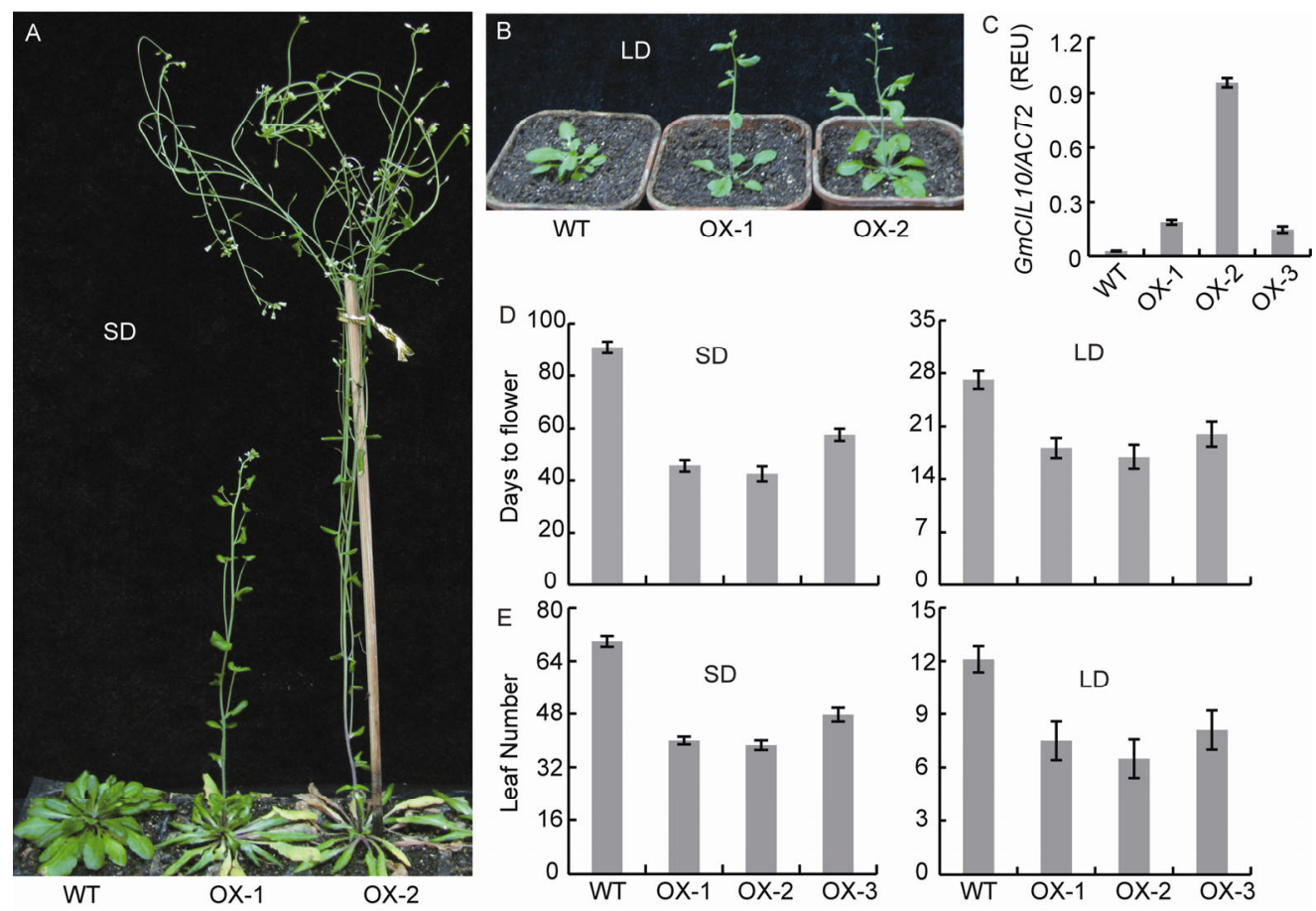

Figure 6 (color online) Flowering phenotypes of Arabidopsis plants grown under SDs or LDs. (A and B) Representative images of indicated plants grown under SDs (A) or LDs (B). (C) Analysis of the GmCIL10 mRNA expression by RT-qPCR in indicated genotypes. (D and E) The statistical analysis of flowering days (D) and rosette leaf number (E) at the time of flowering. The means and standard deviations $(n \geqslant 20)$ are shown. OX-1, OX-2 and OX-3 represents three independent GmCIL10 overexpression transgenic lines respectively.

days to flowering and rosette leaf numbers of three transgenic lines are significant less than those of WT grown under both SDs and LDs (Figure 6D and E), which supports the hypothesis that GmCIL10 acts as a positive regulator of flowering time.

\section{Conclusion}

Arabidopsis CIB1 is a type of bHLH transcription factor, specifically interacting with CRY2 in response to blue light to activate the transcription of FT and trigger flowering initiation. However, it is unclear whether the CRY-CIB signaling mechanism in the regulation of flowering time is evolutionarily conserved in plants species. In this study, we showed that the diurnal mRNA expression rhythm of GmCIL10 under SD photoperiods resembles that of GmCRYla, which is a major regulator of photoperiodic flowering in soybean. In addition, the levels of GmCIL1O transcripts in the unifolioates and trifoliolates under SDs increased gradually with leaf development and then decreased after the initiation of flowering (Figure 4). Such an expression pattern is consistent with that of GmFT2a and GmFT5a, which encode florigens and coordinately control flowering in soybean. Furthermore, ectopic expression of GmCIL1O in Arabidopsis accelerates flowering of transgenic plants grown under both LDs and SDs. Taking together with the observation that GmCIL10 protein localizes in the nucleus of Arabidopsis protoplasts, we argue that GmCIL10 in soybean may function as a transcription factor as CIB1 in Arabidopsis in the aspect of flowering time regulation. However, it remains to be investigated whether GmCRY physically interacts with GmCIL10 to regulate flowering in soybean like CRY2-CIB1 complex does in Arabidopsis in response to blue light. In contrast to Arabidopsis, which is a long-day plant flowering earlier under LDs than in SDs, Soybean in nature is a typical short-day crop flowering earlier under SDs than under LDs. Because the flowering time of soybean is commonly hypersensitive to ambient photoperiods, soybean cultivars in general can only be cultivated in a limited latitudinal zone according to individual photoperiodic flowering habit [22]. Therefore, additional studies are needed to elucidate the role of GmCIL10 as well as other CIB homologs underlying photoperiodic control of soybean flowering time in order to improve the eurytopicity of soybean cultivars in different regions by molecular breeding.

This work was supported in part by the National Natural Science Foundation of China (31371649, 31301346, 31422041) and a Core Research Budget of the Non-profit Governmental Research Institution (Institute of Crop Science, Chinese Academy of Agricultural Sciences).

1 Cashmore AR. Cryptochromes: Enabling Plants and Animals to Determine Circadian Time. Cell, 2003, 114: 537-543

2 Sancar A. Structure and Function of DNA Photolyase and Crypto- 
chrome Blue-Light Photoreceptors. Chem rev, 2003, 103: 2203-2238

3 Lin C, Shalitin D. Cryptochrome structure and signal transduction. Annu revplant biol, 2003, 54: 469-496

4 Liu B, Zuo Z, Liu H, Liu X, Lin C. Arabidopsis cryptochrome 1 interacts with SPA1 to suppress COP1 activity in response to blue light. Genes Dev, 2011, 25: 1029-1034

5 Ahmad M, Cashmore AR. HY4 gene of A. thaliana encodes a protein with characteristics of a blue-light photoreceptor. Nature, 1993, 366: 162-166

6 Guo H, Yang H, Mockler TC, Lin C. Regulation of flowering time by Arabidopsis photoreceptors. Science, 1998, 279: 1360-1363

7 Liu H, Yu X, Li K, Klejnot J, Yang H, Lisiero D, Lin C. Photoexcited CRY2 Interacts with CIB1 to Regulate Transcription and Floral Initiation in Arabidopsis. Science, 2008, 322: 1535-1539

8 Ikeda M, Fujiwara S, Mitsuda N, Ohme-Takagi M. A triantagonistic basic helix-loop-helix system regulates cell elongation in Arabidopsis. Plant Cell, 2012, 24: 4483-4497

9 Liu Y, Li X, Li K, Liu H, Lin C. Multiple bHLH Proteins form Heterodimers to Mediate CRY2-Dependent Regulation of Flowering-Time in Arabidopsis. PLoS Genet, 2013, 9: e1003861

10 Atchley WR, Fitch WM. A natural classification of the basic helixloop-helix class of transcription factors. Proc Natl Acad Sci USA, 1997, 94: 5172-5176

11 Littlewood TD, Evan GI. Helix-loop-helix transcription factors. Oxford University Press New York, 1998.

12 Ledent V, Vervoort M. The basic helix-loop-helix protein family: comparative genomics and phylogenetic analysis. Genome Res, 2001, 11: 754-770

13 Toledo-Ortiz G, Huq E, Quail PH. The Arabidopsis basic/helixloop-helix transcription factor family. Plant Cell, 2003, 15: 17491770

14 Nair SK, Burley SK. Functional genomics: recognizing DNA in the library. Nature, 2000, 404: 715-718

15 Murre C, McCaw PS, Baltimore D. A new DNA binding and dimerization motif in immunoglobulin enhancer binding, daughterless, MyoD, and myc proteins. Cell, 1989, 56: 777-783

16 Ferre-D'Amare A, Pognonec P, Roeder R, Burley S. Structure and function of the b/HLH/Z domain of USF. EMBO J, 1994, 13: 180

17 Meng Y, Li H, Wang Q, Liu B, Lin C. Blue Light-Dependent Inter- action between Cryptochrome2 and CIB1 Regulates Transcription and Leaf Senescence in Soybean. Plant Cell, 2013, 25: 4405-4420

18 Liu H, Liu B, Zhao C, Pepper M, Lin C. The action mechanisms of plant cryptochromes. Trends Plant Sci, 2011, 16: 684-691

19 Xue Z, Zhang X, Lei C, Chen X, Fu Y. Molecular cloning and functional analysis of one ZEITLUPE homolog GmZTL3 in soybean. Mol Biol Rep, 2012, 39: 1411-1418

20 Wu F, Zhang X, Li D, Fu Y. Ectopic Expression Reveals a Conserved PHYB Homolog in Soybean. PLoS ONE, 2011, 6: e27737

21 Huang G, Ma J, Han Y, Chen X, Fu Y. Cloning and Expression Analysis of the Soybean CO-Like Gene GmCOL9. Plant Mol Biol Rep, 2011, 29: 352-359

22 Zhang Q, Li H, Li R, Hu R, Fan C, Chen F, Wang Z, Liu X, Fu Y, Lin C. Association of the circadian rhythmic expression of GmCRY1a with a latitudinal cline in photoperiodic flowering of soybean. PNAS U S A, 2008, 105: 21028-21033

23 Fan C, Wang X, Wang Y, Hu R, Zhang X, Chen J, Fu Y. Genome-Wide Expression Analysis of Soybean MADS Genes Showing Potential Function in the Seed Development. PLoS ONE, 2013, 8: e62288

24 Chen Q, Zhou H, Chen J, Wang X. Using a modified TA cloning method to create entry clones. Anal Biochem, 2006, 358: 120-125

25 Fan C, Wang X, Hu R, Wang Y, Xiao C, Jiang Y, Zhang X, Zheng C, $\mathrm{Fu} \mathrm{Y}$. The pattern of Phosphate transporter 1 genes evolutionary divergence in Glycine max L. BMC Plant Biol, 2013, 13: 48

26 Xiao C, Chen F, Yu X, Lin C, Fu Y. Over-expression of an AT-hook gene, AHL22, delays flowering and inhibits the elongation of the hypocotyl in Arabidopsis thaliana. Plant Mol Biol, 2009, 71: 39-50

27 Yoo S, Cho Y, Sheen J. Arabidopsis mesophyll protoplasts: a versatile cell system for transient gene expression analysis. Nat Protoc, 2007, 2: 1565-1572

28 Kong F, Liu B, Xia Z, Sato S, Kim BM, Watanabe S, Yamada T, Tabata S, Kanazawa A, Harada K. Two coordinately regulated homologs of FLOWERING LOCUS $\mathrm{T}$ are involved in the control of photoperiodic flowering in soybean. Plant Physiol, 2010, 154: 1220-1231

29 Hu R, Fan C, Li H, Zhang Q, Fu Y. Evaluation of putative reference genes for gene expression normalization in soybean by quantitative real-time RT-PCR. BMC Mol Biol, 2009, 10: 93

Open Access This article is distributed under the terms of the Creative Commons Attribution License which permits any use, distribution, and reproduction in any medium, provided the original author(s) and source are credited. 